\title{
Estratégias de avaliação para programas de promoção da saúde com ênfase na atividade física
}

http://dx.doi.org/10.11606/1807-5509201900020301

\author{
Daniel Rogério PETREÇA*/** \\ Paula Fabricio SANDRESCHI* \\ Inês Amanda STREIT* \\ Giovana Zarpellon MAZO* \\ * Universidade do \\ Estado de Santa \\ Catarina, Florianópolis, \\ SC, Brasil. \\ ** Universidade do \\ Contestado, Mafra, SC \\ Brasil.
}

\section{Resumo}

0 objetivo deste estudo é conceituar e diferenciar as dimensões utilizadas nas estratégias de avaliação que podem ser utilizadas em programas públicos de promoção de saúde com ênfase na atividade física. Este estudo caracteriza-se como modelo híbrido de análise conceitual. Para tanto, foram seguidas as fases: teórica; trabalho de campo e analitica final. Foram analisadas as estratégias: (a) RE-AIM - dimensões de alcance, efetividade/eficácia, adoção, implementação e manutenção; (b) modelo proposto por Avedis Donabedian - dimensões de estrutura, processo e resultado, além dos 7 pilares de qualidade; o modelo de Habicht - indicadores de oferta, de utilização, de cobertura e de impacto além de inferências: adequação, plausibilidade e probabilidade. Ao analisar as estratégias de avalição, percebe-se, nomenclaturas distintas, mas pontos convergentes. Ao verificar a dimensão de "Alcance" do RE-AIM ele se assemelha ao de "Cobertura" do Habicht. Os indicadores de "estrutura" e "processo" de Donabedian correspondem aos de oferta de Habicht e de "implementação" do RE-AIM. Ao verificar "resultado" no modelo Donabedian, ele pode ser comparado à "efetividade/eficácia" do RE-AIM e "impacto" no modelo Habicht. Assim, apesar de serem organizados de formas diferentes, as estratégias de avaliação analisadas nesse manuscrito apresentam certa relação nas dimensões, indicadores e elementos, sendo que elas podem ser utilizadas de forma isolada ou como complementares.

Palavras-chave: Atividade Motora; Saúde Pública; Avaliação de Programas; Projetos de Saúde.

\section{Introdução}

A inatividade física é um dos grandes problemas de saúde pública contemporânea ${ }^{1}$, sendo responsável por mais de 3 milhóes de mortes por ano². Isso se deve à relação desse comportamento com muitas condiçôes adversas de saúde, incluindo as principais doenças crônicas não transmissíveis (doenças coronárias, diabetes tipo 2 , alguns tipos de câncer $)^{3}$.

Diante desse quadro, diversas intervençōes de promoçáo da saúde com ênfase em atividade foram implementadas no mundo todo: Estados Unidos Bélgica, Canadá, Grécia, Austrália, Rússia, Inglaterra, Nova Zelândia, Colômbia, Japão ${ }^{4}$ e Brasil ${ }^{5}$. A difusão e o investimento em promoção da saúde com ênfase na atividade física faz com que a avaliação, apesar de não ser frequente no campo da saúde ${ }^{6}$, desperte relevância. Isso ocorre devido à verificação da realidade e às transformaçóes oriundas de uma determinada forma de intervir em saúde; fato que pode contribuir na modificação do quadro da inatividade física e subsidiar as dificuldades enfrentadas na implementação 7 .

De acordo com a AgÊncia Nacional de VigilânCIa Sanitária do Brasil ${ }^{5}$ foi criada uma demanda oriunda da produçáo de conhecimentos em relação a avaliaçóes. Segundo Green e GlasGow ${ }^{8}$, elas devem ser acompanhadas do emprego de construçóes metodológicas; no entanto, podem ter seu papel limitado devido à dificuldade e falta de um quadro de avaliação abrangente e adequado para intervenções específicas.

A avaliação tem como objetivo fornecer informaçôes para órgãos governamentais, privados, financiadores, profissionais e usuários a fim de proporcionar a prestação de contas e comunicar o 
conhecimento obtido9 . Além disso, deve verificar o efeito que o programa teve em ambiente da comunidade, a efetividade e a identificação de oportunidades para melhorias ${ }^{10}$. Isso se torna um desafio dentro de um ambiente natural e complexo ${ }^{11}$.

Muitas vezes a avaliação não é incluída no planejamento da intervenção por ser considerada um processo complexo ${ }^{12}$. Usualmente inicia-se após a intervenção já ter sua implantação estabelecida ${ }^{13}$ uma vez que as questôes avaliativas necessitam uma gama de informações e utilização de diferentes dados para obtençáo de respostas ${ }^{14}$.

Para tanto, estratégias avaliativas foram desenvolvidas. É caso do RE-AIM, proposto pelo Dr. Russell Glasgow, Vogt e Boles ${ }^{15}$, é amplamente utilizado na avaliação de programas de atividade física $^{16-20}$. Outros modelos bastante empregados em açóes da área da saúde são os propostos pela Rede Carmem do Ministério da Saúde ${ }^{7}$ : Modelo Haвicht et al. ${ }^{21}$ e do Dr. Avedis Donabedian ${ }^{22}$.

As estratégias avaliativas auxiliam na decisão de prioridades e são úteis para pesquisadores e profissionais no desenvolvimento ou decisão do processo das intervençôes ${ }^{23}$. São apresentadas na sua composiçâo diferentes dimensôes/indicadores/ elementos de análise de acordo com suas características $^{15,24,25}$.

Apesar da apresentação dessas estratégias, existe dificuldade na enorme diversidade terminológica encontrada nos enfoques teóricos sobre o tema, tanto no que diz respeito às possíveis abordagens quanto no que concerne aos seus atributos ou componentes ${ }^{25}$. Diante disso, observa-se que há necessidade de contextualizar conceitualmente as dimensóes das estratégias de avaliação: RE-AIM, de Donabedian e de Habicht e analisar as relaçóes existentes entre elas, para a utilização de um ou mais estratégias na avaliaçáo de programas públicos de promoçãa de saúde com ênfase na atividade física, de acordo com o contexto que os programas estão inseridos.

Desse modo, apresenta-se como objetivo desse estudo $\mathrm{O}$ objetivo deste estudo é conceituar e diferenciar as dimensôes utilizadas nas estratégias de avaliação que podem ser utilizadas em programas públicos de promoção de saúde com ênfase na atividade física.

\section{Método}

Para atingir esse objetivo a pesquisa foi realizada no modelo híbrido de análise conceitual proposto por SCHWARTZ-BARCOTt e $\mathrm{KIM}^{26}$ o qual interliga análise teórica e observação empírica. Ele foi desenvolvido em três fases: $1^{\circ}$ ) teórica, a qual foi realizada a seleção dos conceitos, busca e revisão da literatura, definição conceitual e operacional dos conceitos, além disto, foram explorados termos presentes em cada dimensão proposta pelas estratégias; $\left.2^{\circ}\right)$ trabalho de campo, na qual foram realizadas observaçôes empíricas de dezembro de 2013 a março 2014. Nesse período foi analisado as dimensões das estratégias utilizando o Programa "Grupo de Estudos da Terceira Idade” (GETI - UDESC), o qual oferece ações em atividade física para aproximadamente 300 idosos da comunidade; $3^{\circ}$ ) analítica final, na qual buscou-se compreender a relação entre as dimensóes da fase teórica inicial (por meio dos estudos dos conceitos e teorias) e as observaçóes empíricas (realizadas no GETI), sendo o produto final a apresentação e comparação conceitual direcionando o desenvolvimento de outras pesquisas.

\section{Resultados e Discussão}

\section{Estratégias de avaliaçáo}

\section{Ferramenta RE-AIM}

A ferramenta RE-AIM foi desenvolvida como um quadro para verificação da consistência em resultados de investigaçóes ${ }^{15}$. Posteriormente, utilizada como instrumento de análise de qualidade de validade interna e externa na organização de revisóes da literatura na promoção da saúde e prevenção das doenças em diferentes contextos ${ }^{27}$.

Além disso, o RE-AIM é utilizado para auxiliar no planejamento de intervençóes com o objetivo de melhorar as chances da reprodutibilidade das açóes em "mundo real" 8,27 . Também possui o objetivo de auxiliar na descrição da pesquisa para a prática, 
estimando assim, o impacto na saúde pública de programas de promoção da saúde, inclusive nos com ênfase em atividade física ${ }^{18,28}$.

É uma ferramenta multidimensional, sendo composto por cinco dimensóes: Alcance (Reach): como fazer para chegar à população-alvo?; Adoção (Adoption): como desenvolver o apoio organizacional?; Implementação (Implementation): como garantir se a intervenção está correta?; Efetividade/Eficácia (Effectiveness): a intervenção é eficaz?; Manutenção (Maintenance): a intervenção continua em longo prazo?

O QUADRO 1 apresenta a definição de cada dimensão seguido dos termos mais utilizados e do nível de avaliação (individual e organizacional) em cada um dos elementos do RE-AIM.

QUADRO 1 - Definição de cada dimensão seguido dos termos mais utilizados e do nível de avaliação em cada um dos elementos do RE-AIM

\begin{tabular}{|c|c|c|}
\hline $\begin{array}{l}\text { Dimensóes do } \\
\text { RE-AIM }\end{array}$ & Definiçóes & \\
\hline ALCANCE & $\begin{array}{l}\text { É o número absoluto, a proporção e a representatividade dos indivíduos } \\
\text { que estão dispostos a participar de uma determinada iniciativa } \\
\text { comparada àqueles que desistem ou àqueles potencialmente elegíveis }\end{array}$ & Individual \\
\hline População alvo & \multicolumn{2}{|l|}{ É a população a qual se deseja atingir. Ex.: idosos, crianças, gestantes, etc. } \\
\hline $\begin{array}{l}\text { Participantes } \\
\text { elegíveis }\end{array}$ & \multicolumn{2}{|c|}{$\begin{array}{l}\text { Sáo aqueles que atingem os critérios de inclusão da intervençáo e assim, estão aptos para } \\
\text { participar. Ex.: idosos independentes fisicamente, }\end{array}$} \\
\hline Recrutamento & \multicolumn{2}{|c|}{ É a estratégia de busca por participantes elegíveis. Ex.: panfletagem, anúncios, etc } \\
\hline Taxa de participação & \multicolumn{2}{|c|}{$\begin{array}{l}\text { O número de participantes dividido pelo número total de pessoas que são elegíveis para } \\
\text { a participaçáo. }\end{array}$} \\
\hline Representatividade & \multicolumn{2}{|c|}{ O quanto, em números, os participantes representam a população-alvo. } \\
\hline ADOÇÃO & $\begin{array}{l}\text { É o número absoluto, a proporção e a representatividade das organizaçóes } \\
\text { e dos agentes de intervenção que estáo dispostos a iniciar um programa. }\end{array}$ & $\begin{array}{l}\text { Organizacional } \\
\text { e individual }\end{array}$ \\
\hline & \multicolumn{2}{|c|}{$\begin{array}{l}\text { Nível organizacional: organizaçóes onde se deseja implantar a intervenção. Ex.: centros } \\
\text { de saúde de Florianópolis. } \\
\text { Nível individual: população profissional a qual se deseja atingir para trabalhar na } \\
\text { intervenção. Ex.: Profissionais de Educação Física. }\end{array}$} \\
\hline $\begin{array}{l}\text { Participantes } \\
\text { elegíveis }\end{array}$ & \multicolumn{2}{|c|}{$\begin{array}{l}\text { Nível organizacional: organizaçóes que atingem os critérios de inclusão e assim, estão } \\
\text { aptos a receber a intervenção. Ex.: centros de saúde da região central } \\
\text { Nível individual: aqueles que atingem os critérios de inclusão da intervenção e assim, } \\
\text { estão aptos para atuar. Ex.: Profissional de Educação Física Especialista. }\end{array}$} \\
\hline Recrutam & \multicolumn{2}{|c|}{$\begin{array}{l}\text { Nível organizacional: estratégia de busca por organizaçóes elegíveis. Ex.: contato } \\
\text { telefônico, e-mail, etc. } \\
\text { Nível individual: estratégia de busca por profissionais elegíveis. Ex.: Edital de seleção }\end{array}$} \\
\hline Taxa de participação & \multicolumn{2}{|c|}{$\begin{array}{l}\text { Nível organizacional: número de organizaçóes participantes divididos pelo número total } \\
\text { organizações que são elegíveis para a participação. } \\
\text { Nível individual: número de profissionais participantes dividido pelo número de } \\
\text { profissionais que são elegíveis para participação }\end{array}$} \\
\hline Representatividade & \multicolumn{2}{|c|}{$\begin{array}{l}\text { Nível organizacional: o quanto, em números, as organizaçóes participantes representam } \\
\text { as organizaçóes-alvo. } \\
\text { Nível individual: o quanto, em números, os profissionais participantes representam os } \\
\text { profissionais-alvo. }\end{array}$} \\
\hline IMPLEMENTAÇÃO & $\begin{array}{l}\text { Nível organizacional: refere-se à fidelidade dos agentes de intervenção } \\
\text { aos vários elementos de um protocolo de intervenção. Isso inclui a } \\
\text { consistência no fornecimento, conforme previsto, o tempo e o custo da } \\
\text { intervenção. Nível individual: refere-se à medida que os participantes } \\
\text { utilizam as estratégias da intervenção. }\end{array}$ & $\begin{array}{l}\text { Organizacional } \\
\text { e individual }\end{array}$ \\
\hline
\end{tabular}


Fonte: ALMEIDA, BRITO e ESTABROOKS 29 ; RE-AIM ${ }^{30}$.

Continuação

QUADRO 1 - Definição de cada dimensão seguido dos termos mais utilizados e do nível de avaliação em cada um dos elementos do RE-AIM

\begin{tabular}{|c|c|c|}
\hline $\begin{array}{c}\text { Dimensóes do } \\
\text { RE-AIM } \\
\end{array}$ & Definiçóes & $\begin{array}{c}\text { Nível de } \\
\text { avaliaçáo }\end{array}$ \\
\hline Custo-efetividade & \multicolumn{2}{|c|}{$\begin{array}{l}\text { É a soma de todos os custos gerados em cada dimensáo dividido pela unidade de medida } \\
\text { do objetivo geral da intervençáo. Ex.: se o objetivo geral é a perda de massa corporal, o } \\
\text { custo efetividade seria que cada kg perdido custa } \mathrm{R} \$ 20 \text {. }\end{array}$} \\
\hline Custo global & \multicolumn{2}{|c|}{$\begin{array}{l}\text { É a soma de todos os custos gerados em cada dimensáo dividido pelo número de } \\
\text { participantes. Ex.: cada participante tem um custo de } \mathrm{R} \$ 50 \text { mensais. }\end{array}$} \\
\hline Elementos centrais & \multicolumn{2}{|c|}{$\begin{array}{l}\text { Principais açóes que devem ser feitas para o sucesso da intervenção. Avaliar a } \\
\text { implementaçáo é identificar os elementos centrais }\end{array}$} \\
\hline Modelo lógico & \multicolumn{2}{|c|}{$\begin{array}{l}\text { Estratégia que permite a compreensão da iniciativa e seu monitoramento para que haja } \\
\text { maior controle na gestão. Auxilia na descrição da implementação. }\end{array}$} \\
\hline $\begin{array}{l}\text { EFET } \\
\text { EFIC }\end{array}$ & $\begin{array}{l}\text { É o impacto de uma intervenção sobre os desfechos incluindo } \\
\text { qualidade de vida, efeitos negativos e os resultados econômicos. }\end{array}$ & Individual \\
\hline Fatore & \multicolumn{2}{|c|}{$\begin{array}{l}\text { Algum fator fora do esperado que ocorreu com a intervenção. Ex.: uma ampla divulgação } \\
\text { de um programa de atividade física para idosos faz com que os mesmos saiam mais de } \\
\text { casa, o que aumenta o número de quedas nessa populaçáo. }\end{array}$} \\
\hline Objetivo & \multicolumn{2}{|l|}{ É mensurável. Se a mensuração não for possível, ele não está claro. } \\
\hline Meta & \multicolumn{2}{|l|}{ Propõe uma direção, um horizonte, mas não é palpável. } \\
\hline MANUTENÇÃO & $\begin{array}{l}\text { Nível organizacional: medida que um programa ou política torna- } \\
\text { se institucionalizado ou parte das práticas de rotina e políticas } \\
\text { organizacionais. Nível individual: a manutenção tem sido definida } \\
\text { como os efeitos benéficos em longo prazo (seis ou mais meses após o } \\
\text { término da intervençáo). }\end{array}$ & $\begin{array}{l}\text { Organizacional } \\
\text { e individual }\end{array}$ \\
\hline
\end{tabular}

Fonte: Almeida, Brito e Estabrooks ${ }^{29}$; RE-AIM ${ }^{30}$.

O RE-AIM pode ser utilizado como ferramenta de avaliação e planejamento. Para tanto, a FIGURA 1 demonstra um exemplo (intervençáo de ginástica para idosos com objetivo de aumentar nível de atividade física, qualidade de vida e aptidão física) da sua utilização neste processo.

O RE-AIM, tem um enfoque na valorização $\mathrm{da}$ validade interna e externa por meio das respostas das suas dimensões, que em resumo são: "alcance" refere-se à porcentagem e características dos indivíduos que receberam a intervenção; "efetividade/eficácia" refere-se ao impacto da intervenção, incluindo o previsto, bem como resultados imprevistos; "adoção" diz respeito à porcentagem e representatividade das definiçóes que adotam a intervenção; "implementação" referese à consistência e custo de entrega da intervenção; e "manutenção" refere-se a sustentabilidade a longo prazo, tanto a definição e os níveis individuais ${ }^{15,30}$.

Desde a sua concepçáo, a ferramenta RE-AIM continua a evoluir sendo traduzido e adaptado culturalmente para a realidade brasileira em $2013^{29}$. A sua aplicaçáo ocorre em uma variedade de esferas, com inúmeras populaçóes, incluindo o envelhecimento, rastreamento de câncer, mudança alimentar, política de saúde pública, saúde da mulher, entre outros ${ }^{30}$. Sendo que em revisão sistemática, GAGLIO et al. ${ }^{18}$ verificaram em estudos utilizando a ferramenta, maior frequência do uso na atividade física, obesidade e controle da doença. Ainda, são localizados diversos estudos de avaliação de programas de atividade física por meio desta ferramenta ${ }^{17,19,20,31,32}$.

Segundo Belza, Toobert e Glasgow ${ }^{33}$ para maximizar o impacto de uma intervenção, a ferramenta RE-AIM deve ser utilizada com base nos cincos elementos em conjunto, sendo que, a apresentação de fragilidade em qualquer uma das dimensóes pode afetar negativamente o impacto e a implantaçáo.

Dessa forma, apesar de alguma sobreposição, cada um dos elementos foi projetado para ser analisado em harmonia, não dispondo de um mais importante, 
ocorrendo modificaçóes dependentes ao objetivo predeterminado por cada programa.

O RE-AIM, é uma ferramenta conceitual e segundo King, Glasgow, Leeman-Castillo ${ }^{34}$ ele não possui uma estrutura definida no fornecimento de orientaçóes específicas de dados e como os coletá-los. Para tanto, a obtenção de resultados de cada uma das dimensóes e suas características deve responder à questão problema de cada elemento definindo o que medir, como medir e com quem medir utilizando diversas técnicas de abordagem, tanto qualitativas e quantitativas em modelos mistos de estudos o que pode representar limitaçóes em relação ao seu uso.

\section{PLANEJAMENTO UTILIZANDO O MODELO RE-AIM} Intervençáo de prática de ginástica para idosos

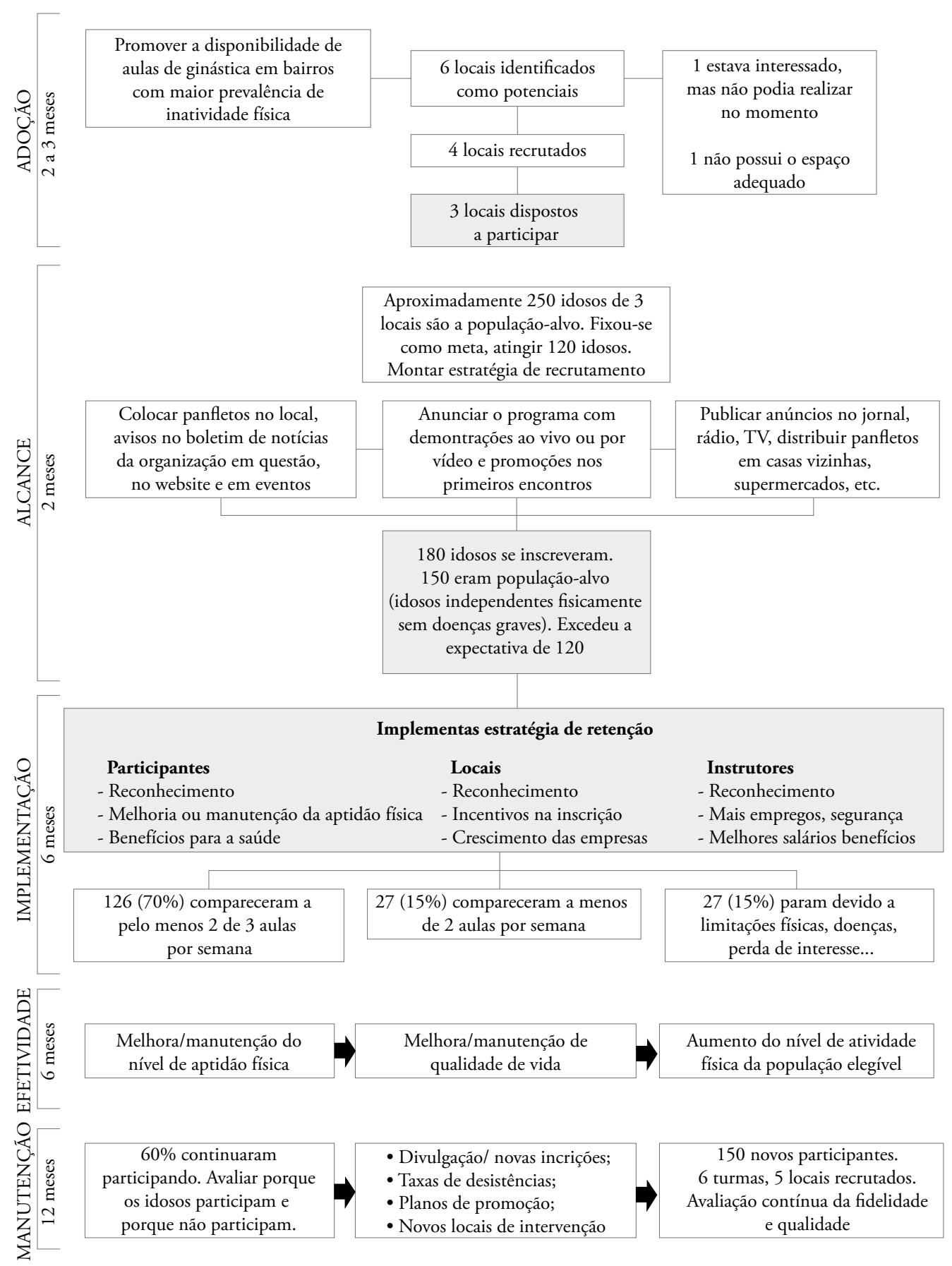

FIGURA 1 - Exemplo da utilização do Modelo RE-AIM na avaliação e planejamento de intervenções.

Fonte: Adaptado RE-AIM ${ }^{30}$. 


\section{Modelo Habicht}

O modelo de avaliação proposto por НABıcHT et al. ${ }^{21}$ tem enfoque voltado à saúde pública e propóe dois eixos. O primeiro eixo refere-se aos tipos de indicadores necessários para avaliação. $\mathrm{Ou}$ seja, quâo longe se deseja ir na avaliação segundo os indicadores de:

- Oferta: verifica se os serviços estão disponíveis, se são acessíveis à população e se a qualidade é adequada.

- Utilização: verifica se os serviços estão sendo utilizados pela população.

- Cobertura: verifica se a utilização resultará em uma dada cobertura populacional. A cobertura é uma medida particularmente útil, representando a interface entre serviços ou

- Oferta de atividades (o processo gerencial) com a população (o quadro epidemiológico).

- Impacto: avalia se a cobertura alcançada pode levar a um impacto sobre o desempenho, o comportamento ou a saúde, e, ainda, se houve um resultado favorável.

Esses indicadores estão relacionados ao que se quer medir e avaliar. Nesse eixo a população- alvo é o grupo populacional a ser atingido pela intervençấo ${ }^{7,35}$.

A FIGURA 2 demonstra um exemplo de utilizaçâo do modelo Habicht em uma intervenção de ginástica para idosos com objetivo de aumentar nível de atividade física, qualidade de vida e aptidão física.

O segundo eixo do modelo Habicht referese ao tipo de inferência causal (adequação, plausibilidade ou probabilidade) e ao grau de certeza necessária para a tomada de decisão. O nível de inferência depende do tipo de estudo empregado para a avaliação ${ }^{35}$ e está diretamente relacionado ao estágio de desenvolvimento do programa e seus recursos disponíveis ${ }^{7}$. Tanto as avaliaçóes de processo (oferta, utilização e cobertura) quanto as de impacto podem utilizar inferência de adequação, plausibilidade ou probabilidade ${ }^{7}$.

A FIGURA 2 apresenta os níveis de inferência para a avaliação, o seu desempenho e impacto, conforme o modelo Habicht.

O modelo de Habitch tem enfoque voltado para a saúde pública e os indicadores de oferta, utilização, cobertura e impacto pode passar por avaliação pelo grau de inferência (adequação, plausibilidade e probabilística) necessário para responder a pergunta avaliativa.

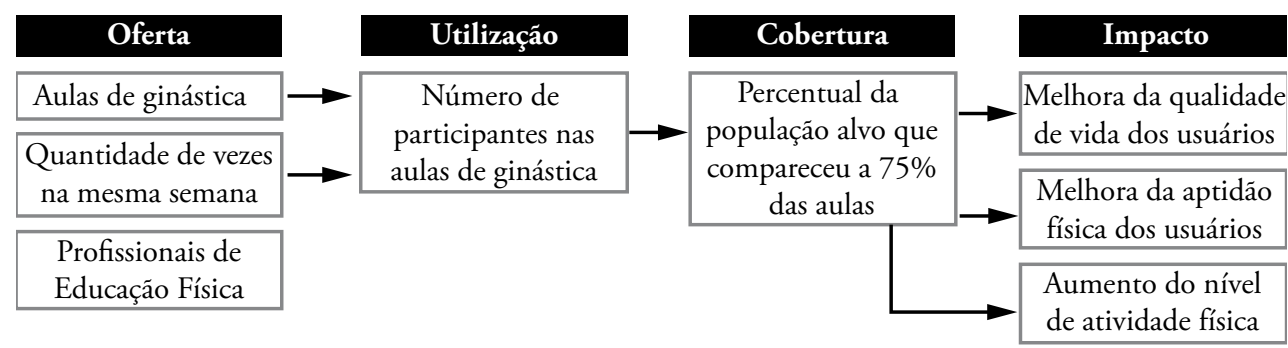

FIGURA 2 - Exemplo da utilização do modelo Habicht em uma intervenção de atividade física.

QUADRO 3 - Níveis de inferência para a avaliação de intervenção, conforme o modelo Habicht

\begin{tabular}{|c|c|c|}
\hline \multicolumn{3}{|r|}{ Níveis de inferência para a avaliaçáo } \\
\hline \multirow{2}{*}{ 胥 } & Desempenho & $\begin{array}{l}\text { Avalia indicadores de oferta, utilização e cobertura, além de verificar se as atividades } \\
\text { foram desenvolvidas como planejadas no início da intervenção }{ }^{36} \text {. A avaliaçáo é realizada } \\
\text { com os profissionais e beneficiários }{ }^{7} \text {. Envolve estudos transversais e longitudinais. }\end{array}$ \\
\hline & Impacto & $\begin{array}{l}\text { Avalia indicadores de saúde e comportamento }{ }^{36} \\
\text { Significa o efeito de uma intervenção ou programa sobre sua população alvo. Verifica } \\
\text { se a mudança observada na saúde ou no comportamento é na direção e magnitude } \\
\text { esperadas. Envolve estudos transversais e longitudinais. }\end{array}$ \\
\hline
\end{tabular}


QUADRO 3 - Níveis de inferência para a avaliação de intervenção, conforme o modelo Habicht

\begin{tabular}{|c|c|c|}
\hline \multicolumn{3}{|r|}{ Níveis de inferência para a avaliaçáo } \\
\hline \multirow{2}{*}{ 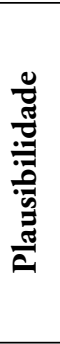 } & Desempenho & $\begin{array}{l}\text { Avalia indicadores de oferta, utilização e cobertura. Responde se o grupo de intervenção } \\
\text { parece ter melhor desempenho do que o grupo controle }{ }^{36} \text {. Avalia as atividades do } \\
\text { programa por meio dos profissionais que implementaram e beneficiários. Envolve } \\
\text { estudos transversais, longitudinais e longitudinais-controle. }\end{array}$ \\
\hline & Impacto & $\begin{array}{l}\text { Avalia indicadores de saúde e de comportamento em beneficiários e populaçáo-alvo } \\
\text { e verifica se as mudanças nos parâmetros de saúde ou comportamentais parecem ser } \\
\text { mais benéficos no grupo de intervençáo do que no grupo controle }{ }^{36} \text {. Abrange estudos } \\
\text { transversais, longitudinais, longitudinais-controle caso-controle. }\end{array}$ \\
\hline \multirow{2}{*}{ 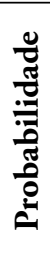 } & Desempenho & $\begin{array}{l}\text { Avalia indicadores de oferta, utilização e cobertura, verificando as atividades do programa } \\
\text { por meio dos profissionais e beneficiários. Verifica se o grupo experimental tem melhor } \\
\text { desempenho que o grupo controle. Abrange estudos longitudinais, caso-controle }{ }^{7} \text {. }\end{array}$ \\
\hline & Impacto & $\begin{array}{l}\text { Avalia indicadores de saúde e de comportamento por meio dos beneficiários do } \\
\text { programa e verifica se a mudança na saúde e no comportamento é melhor no grupo } \\
\text { intervenção do que no controle. Abrange estudos longitudinais, caso-controle }{ }^{7} \text {. }\end{array}$ \\
\hline
\end{tabular}

Fonte: Pedraza ${ }^{36}$; Brasil .

Avaliações de adequação de uma intervenção respondem se as mudanças esperadas aconteceram ou não. Esse grau de inferência depende da comparação entre o desempenho (processo) ou o impacto do programa/intervenção e critérios de adequação estabelecidos a priori ${ }^{7}$. As avaliaçóes de adequação não necessitam da utilização de grupo controle se os resultados forem comparados com critérios preestabelecidos (por exemplo, 90\% dos indivíduos engajados em atividade física regular).

O segundo tipo de inferência é a de plausibilidade, diferente da adequação, ela tenta reduzir fatores externos (fatores de confusão) que podem ter causado os resultados observados ${ }^{7}$. Esse tipo de avaliaçáo requer que sejam descartados os efeitos de fatores externos ao programa, havendo, portanto, a necessidade de um grupo de comparação (grupo controle) para ajuste de fatores de confusáo, por meio de estudos "quase experimentais" 35.

Em outra perspectiva, as avaliações de probabilidade, conduzidas exclusivamente por meio de ensaios randomizados, priorizam a validade interna da avaliaçáo: sendo particularmente adequados para verificar o efeito de intervençóes cuja cadeia causal entre a intervenção e o resultado é curta.

\section{Modelo Donabedian}

Proposto na década de 60 por Avedis Donabedian, esse modelo é utilizado na avaliação da qualidade dos serviços de saúde sendo escrito em $1966^{37}$. No seu modelo, Donabedian identificou três dimensóes ${ }^{22}$ que podem ser utilizadas para avaliar qualidade dos cuidados de saúde, sendo elas:

- Estrutura: Refletem as condições de área física, tecnologia apropriada, recursos humanos, medicamentos, acesso a normas de avaliação e manejo dos usuários. Identificam as condiçóes sob as quais as atividades da intervenção são oferecidas.

- Processo: Indicam o que é realmente oferecido aos usuários em termos de cuidado. Apontam o que os profissionais fazem, em termos de coleta de história, exame físico, exames complementares solicitados, tratamento e acompanhamento a curto, médio ou longo prazo.

- Resultado: Reflete o quanto o usuário do serviço teve sua queixa ou problema resolvido, em decorrência da intervenção após determinado período de tempo. Os indicadores de resultado precisam ser avaliados levando em conta o nível de complexidade do serviço e sua adequação para resolver o tipo de problemática apresentada pelo usuário. Ha problemas cuja complexidade pode ser atendida satisfatoriamente no nível primário de atenção. Outros exigirão tecnologia e recursos humanos de maior complexidade.

A FIGURA 3 exemplifica a utilização do modelo Donabedian em uma intervenção de atividade física, em suas diferentes dimensões de avaliação. 


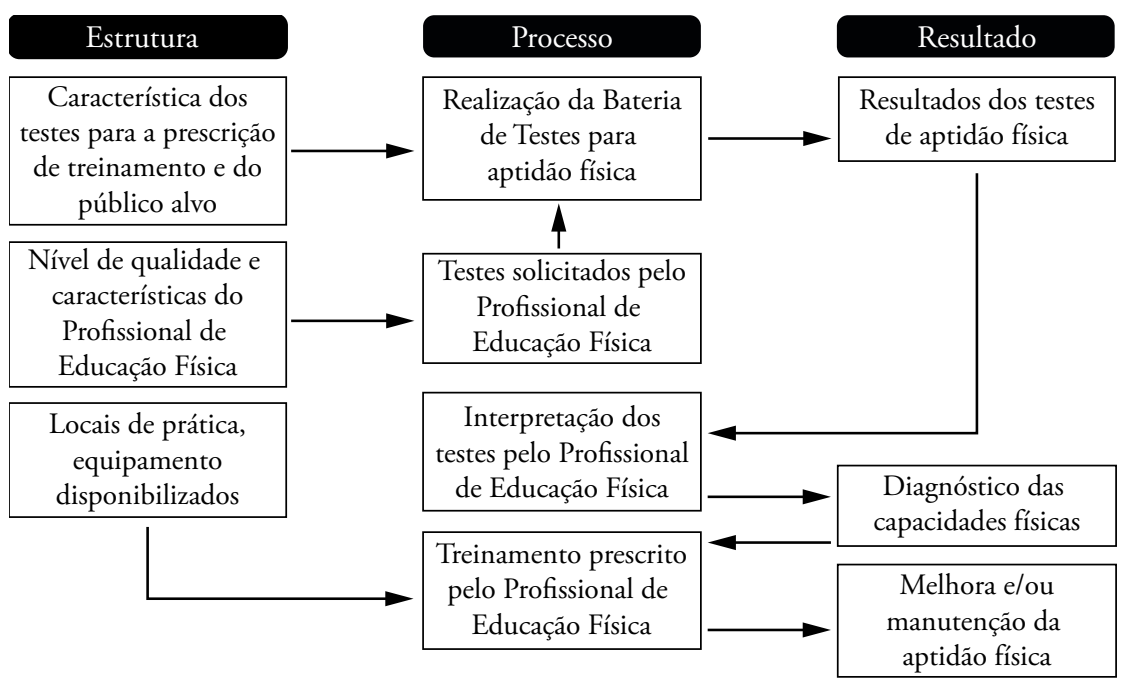

FIGURA 3 - Exemplo da utilização do modelo Donabedian em uma intervenção de atividade física.

O modelo Donabedian, por meio do material intitulado "Avaliaçáo da Qualidade da Assistência Médica", tornou-se um dos métodos mais citados do século 20 ao se relacionar com a saúde pública ${ }^{38}$.

$\mathrm{Na}$ apresentação do seu modelo, Donabedian tem um enfoque na avaliação de serviços e na qualidade, que pode ser influenciada pela variaçáo de acordo com a ótica empregada ${ }^{22}$. Ou seja, percepções diferentes podem estabelecer a qualidade como a capacidade de chegar a metas desejáveis por meios legítimos ${ }^{22}$.

Por exemplo, profissionais de Educação Física julgam a qualidade sob critérios técnicos relacionados à prescrição de treinamento, enquanto o usuário/ aluno terá a ótica no interesse do bem-estar que pode alcançar por meio da prática fundamentada em fatores pessoais e culturais, tendo como componentes: a estrutura (situação física), o processo (implementação) e os resultados (resolução de acordo com o objetivo proposto).

Segundo a Organização Mundial da Saúde ${ }^{39}$ indicadores de estrutura e processo são fundamentais para monitorar e avaliar estratégias de promoção da Atividade Física. Para Donabedian ${ }^{40}$, a estrutura é um elemento muito importante, pois sua construção reflete no desenvolvimento dos processos e seus consequentes resultados. Já o processo, é o caminho mais direto para avaliação da qualidade ${ }^{41}$ e os resultado ${ }^{40}$ possuem a característica de refletir os efeitos de todos os insumos, podendo servir de indicador para a avaliação indireta, tanto da estrutura quanto do processo.

Além disso, a partir das dimensões de estrutura, processo e resultado, Donabedian ${ }^{42}$ propôs uma ampliação dos critérios a serem utilizados, os quais são conhecidos como "7 pilares da qualidade".
1) Eficácia (Efficacy): capacidade de atendimento, em melhor nível, para melhorar a saúde.

2) Efetividade (Effectiveness): nível em que a melhoria da saúde definidos são realizados.

3) Eficiência (Efficiency): capacidade de obter a maior melhoria da saúde ao menor custo possível.

4) Otimização (Optimality): balanço mais vantajosa de custos e benefícios.

5) Aceitabilidade (Acceptability): preferências do usuário em relação à acessibilidade, a relação entre profissional-usuário, as amenidades, efeitos e custos dos cuidados.

6) Legitimidade (Legitimacy): preferências sociais relativas a todas as anteriores.

7) Equidade (Equity): equidade na distribuição do cuidado e seus efeitos sobre a saúde.

\section{Estratégias de avaliação de intervençóes e relaçóes quanto às suas dimensóes}

As estratégias apresentadas nesse estudo podem conter ferramentas úteis no planejamento e avaliação de intervenções. Porém, deve ser ressaltada a limitação da utilização dada pela percepção do gestor na construção metodológica na implantação de programas de promoção de saúde. Além disso, os profissionais de saúde devem levar em conta também as preferências dos usuários, bem como as preferências sociais na avaliação e garantia da qualidade ${ }^{42}$.

Em resumo, o RE-AIM tem como base os fatores individuais e organizacionais por meio das dimensóes de Alcance, Efetividade/ 
Eficácia, Adoção, Implementação e Manutenção, equilibrando as validades internas e externas ${ }^{15}$. O modelo Donabedian apresenta três dimensōes de avaliação da qualidade dos cuidados: estrutura, processo e resultado ${ }^{37}$, ampliando-se posteriormente para os "7 pilares da qualidade": eficácia, efetividade, eficiência, otimizaçáo, aceitabilidade, legitimidade ${ }^{42}$. $\mathrm{O}$ modelo de Habicht propóe dois eixos, o primeiro eixo define indicadores de oferta, de utilização, de cobertura e de impacto e o segundo é composto por três tipos de inferências: adequação, plausibilidadee probabilidade ${ }^{21}$.

Ao verificar as 3 estratégias apresentadas, podese perceber, nomenclaturas distintas, mas pontos convergentes na avaliação de intervençôes de promoção da saúde, apesar de serem organizadas de formas diferentes, apresentam certa relação nas dimensóes (FIGURA 4).

Ao verificar a dimensão de "Alcance" do REAIM ele se assemelha ao de "Cobertura" do Habicht. Os indicadores de "estrutura" e "processo" de Donabedian correspondem aos de oferta de Habicht e de "Implementação" do RE-AIM.

Ao verificar "resultado" no modelo Donabedian, ele pode ser comparado à "efetividade/eficácia" do RE-AIM e "impacto" no modelo Habicht, além disso, os 7 pilares para avaliação da qualidade propostos pelo primeiro autor podem representar questôes ligadas a "efetividade/eficácia" do RE-AIM

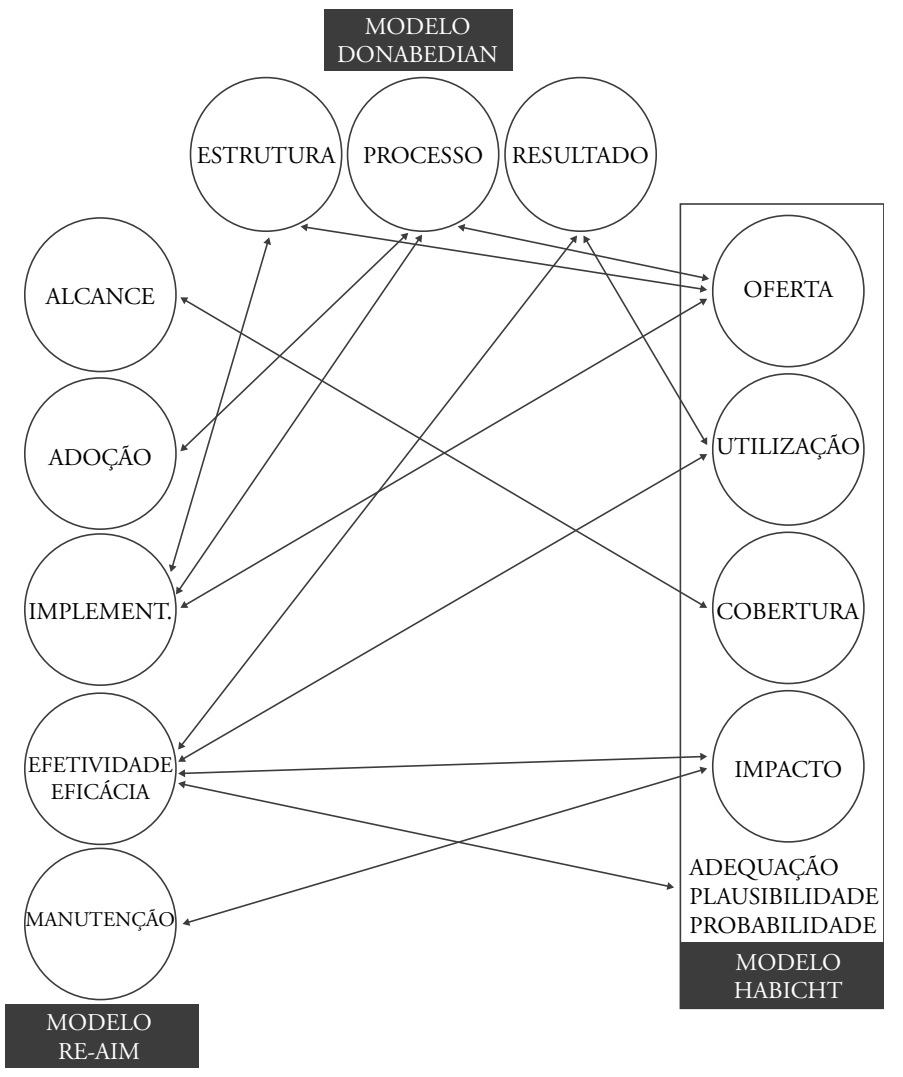

FIGURA 4 - Relações entre os modelos Donabedian, Habicht e RE-AIM.

Por fim, a intenção deste estudo não foi delegar nível de qualidade das estratégias de avaliação: REAIM, Modelo de Donabedian e Modelo de Habicht, mas sim apresentar e conceituar os principais termos utilizados de acordo com suas dimensóes.

Foi verificado que os modelos podem se completar, sendo que a escolha da utilizaçáo das dimensóes, pode acontecer pela análise do pesquisador ou gestor de acordo com as características da intervenção em atividade física e as respostas que queira obter de acordo com as necessidades.

Destaca-se como fundamental a avaliação da promoção da saúde com ênfase em atividade física para um melhor entendimento das relaçóes entre gestores, profissionais e usuários subsidiando mecanismos para manutenção e melhoria das intervençóes. Portanto, independentemente da estratégia de avaliaçáo utilizada, esse procedimento é indispensável e deveria ser organizado desde o princípio da criaçáo da intervenção. 


\section{Abstract \\ Evaluation Strategies for health promotion programs with Physical Activity emphasis}

The objective of this study is to conceptualize and differentiate the dimensions used in the evaluation strategies that can be used in public health promotion programs with physical activity emphasis. This study is characterized as a hybrid model of conceptual analysis. For that, the following phases were followed: theoretical; field work and final analysis. The following strategies were analyzed: (a) REAIM - dimensions of reach, effectiveness/effectiveness, adoption, implementation and maintenance; (b) model proposed by Avedis Donabedian - dimensions of structure, process and result, besides the 7 quality pillars; the Habicht model - indicators of provision, utilization, coverage and impact as well as inferences: adequacy, plausibility and probability. When analyzing the evaluation strategies, one can perceive, distinct nomenclatures, but convergent points. In verifying the scope of RE-AIM "Reach" it resembles that of "coverage" of the Habicht. The Donabedian "structure" and "process" indicators correspond to the Habicht offer and the "implementation" of the RE-AIM. By checking "result" in the Donabedian model, it can be compared to the "effectiveness/effectiveness" of the RE-AIM and "impact" in the Habicht model. Thus, despite being organized in different ways, the evaluation strategies analyzed in this manuscript have a certain relation in the dimensions, indicators and elements, and they can be used in isolation or as complementary.

KeYwords: Motor Activity; Public Health; Program Evaluation.

\section{Agradecimentos}

Ao Conselho Nacional de Desenvolvimento Científico e Tecnológico (CNPq) pela bolsa de produtividade concedida à GZ Mazo, orientadora desse estudo.

\section{Referências}

1. Kohl HW 3rd, Craig CL, Lambert EV, et al. The pandemic of physical inactivity: global action for public health. Lancet. 2012;380(9838):294-305.

2. Pratt M, Sarmiento OL, Montes F, et al. The implications of megatrends in information and communication technology and transportation for changes in global physical activity. Lancet 2012; 380(9838):282-93.

3. Lee IM, Shiroma EJ, Lobelo F, Puska P, Blair SN, Katzmarzyk PT. Effect of physical inactivity on major non-communicable diseases worldwide: an analysis of burden of disease and life expectancy. Lancet. 2012;380(9838):219-29.

4. Heath GW, Parra DC, Sarmiento OL, Andersen LB, Owen N, Goenka S. Evidence-based intervention in physical activity: lessons from around the world. Lancet. 2012;380(9838):272-81.

5. Brasil. Ministério da Saúde. Secretaria de Vigilância em Saúde. Avaliação de efetividade de programas de atividade física no Brasil. Brasília: Ministério da Saúde; 2011.

6. Hoehner CM, Soares J, Parra PD, et al. Physical activity interventions in Latin America: a systematic review. Am J Prev Med. 2008;34(3):224-33.

7. Brasil. Ministério da Saúde. Secretaria de Vigilância em Saúde. Departamento de Análise de Situação de Saúde. Guia metodológico de avaliação e definiçáo de indicadores: doenças crônicas não transmissíveis e Rede Carmem. Brasília: Ministério da Saúde; 2007.

8. Green LW, Glasgow RE. Evaluating the relevance, generalization, and applicability of research issues in external validation and translation methodology. Eval Health Prof. 2006;29(1):126-53.

9. Sparks M. Why evaluate? Professional development in health promotion, 2004. Healthlink The Health Promotion Journal of the ACT Region. 2004;2. 
10. Round R, Marshall B, Horton K. Planning for effective health promotion evaluation. Melbourne: Victorian Government Department of Human Services; 2005.

11. Souza EM. Evaluation methods in health promotion programmes: the description of a triangulation in Brazil. Ciênc Saúde Coletiva. 2010;15(5):2521-32.

12. Rootman I, Goodstadt M, Hyndman B, et al. A framework for health promotion evaluation. WHO Reg Publ Eur Ser. 2001;(92):7-38.

13. Bamberger M, Rugh J, Mabry, L. Realworld evaluation: working under budget, time, data and political constraints. Thousand Oaks: Sage; 2006.

14. Harger-Forde S. Community Development Evaluation Research: Literature Review of Evaluation Methods \& Methodologies. Auckland: Community Waitakere; 2012.

15. Glasgow RE, Vogt TM, Boles SM. Evaluating the public health impact of health promotion interventions: the RE-AIM framework. Am J Public Health. 1999;89(9):1322-7.

16. Benedetti TRB, Schwingel A, Chodzko-Zajko, W, Meurer ST, Brito FA, Almeida FA. RE-AIM: uma proposta de avaliaçáo de programas de atividade física. Kairós. 2014;17(2):295-314.

17. Phillips SM, Alfano CM, Perna FM, Glasgow RE. Accelerating translation of physical activity and cancer a more integrated and collaborative approach Survivorship research into practice: recommendations for a more integrated and collaborative approach. Cancer Epidemiol Biomarkers Prev. 2014;23(5):687-99.

18. Gaglio B, Glasgow RE. Evaluation approaches for dissemination and implementation research 2012. In: Brownson RC, Colditz GA, Proctor EK, editors. Dissemination and implementation research in health: translating science to practice. New York: Oxford University Press; 2012.

19. Jauregui E, Pacheco A, Saavedra F. Programs promoting physical activity among mexicans. Int J Exer Sci [Internet]. 2012 [citado 30 jul 2019];6(2). Disponível em: https://digitalcommons.wku.edu/ijesab/vol6/iss2/26/.

20. Austin G, Bell T, Caperchione C, Mummery WK. Translating Research to Practice: using the RE-AIM framework to examine an evidence-based physical activity intervention in primary school settings. Health Promot Pract. 2011;12(6):932-41.

21. Habicht JP, Victora CG, Vaughan JP. Evaluation designs for adequacy, plausibility and probability of public health programme performance and impact. Int J Epidemiol. 1999;28(1):10-8.

22. Donabedian A. La calidade de la atención médica: definición y métodos de evaluación. Ciudad de México: La Prensa Médica Mexicana; 1984.

23. Glasgow RE. Evaluation of theory-based interventions 2002. In: Glanz K, Rimer BK, Viswanath K, editors. Health Behavior and Health Education: theory, research and practice. San Francisco: Jossey-Bass; 2002.

24. Knuth AG, Malta DC, Cruz DKA, et al. Rede nacional de atividade física do Ministério da Saúde: resultados e estratégias avaliativas. Rev Bras Ativ Fís Saúde. 2010;15(4):229-33.

25. Silva LMV, Formigli VLA. Avaliação em saúde: limites e perspectivas. Cad Saúde Pública. 1994;10(1):80-91.

26. Schwartz-Barcott D, Kim HS. An expansion and elaboration of the hybrid model of concept development. In: Rodgers BL, Knalf KA. Concept development in nursing: foundations, techiniques, and applications. 2nd ed. Philadelphia: Saunders; 2000.

27. Klesges LM, Estabrooks PA, Dzewaltowski DA, Bull SS, Glasgow RE. Beginning with the application in mind: designing and planning health behavior change interventions to enhance dissemination. Ann Behav Med. 2005;29(Suppl):66-75.

28. Dzewaltowski DA, Estabrooks PA, Glasgow RE. The future of physical activity behavior change research: what is needed to improve translation of research into health promotion practice? Exerc Sport Sci Rev. 2004;32(2):57-63.

29. Almeida FA, Brito FA, Estabrooks PA. Modelo RE-AIM: tradução e adaptação cultural para o Brasil. REFACS. 2013;1(1):6-16.

30. RE-AIM. What is RE-AIM [Internet]. Blacksburg: Virginia Tech; 2014 [citado 30 jul 2019]. Disponível em: http://www. re-aim.org/about/what-is-re-aim/.

31. De Meij JS, Chinapaw MJ, Kremers SP, Van der Wal MF, Jurg ME, Van Mechelen W. Promoting physical activity in children: the stepwise development of the primary school-based JUMP-in intervention applying the RE-AIM evaluation framework. Br J Sports Med. 2010;44(12):879-87.

32. Bryant LL, Altpeter M, Whitelaw NA. Evaluation of health promotion programs for older adults: na introduction.J Appl Gerontol [Internet]. 2006 [citado 30 jul 2019];25(3). Disponível em: https://journals.sagepub.com/doi/10.1177/0733464806288562.

33. Belza B, Toobert DJ, Glasgow RE. RE-AIM for program planning: overview and applications. Washington: National Council on Aging; 2007.

34. King DK, Glasgow RE, Leeman-Castillo B. Reaiming RE-AIM: using the model to plan, implement, and evaluate the effects of environmental change approaches to enhancing population health. Am J Public Health. 2010;100(11):2076-84.

35. Santos IS. Avaliação do impacto de programas nutricionais. Rev Nutr. 2009;22(1):141-50. 
36. Pedraza DF. Epidemiologia e avaliação de serviços de saúde. RBPS. 2011;24(2):151-8.

37. Donabedian A. Evaluating the quality of medical care. 1966. Milbank Q. 2005;83(4):691-729.

38. Sunol R. Avedis Donabedian. Int J Qual Health Care. 2000;12(6):451-4.

39. World Health Organization. Global recommendations on physical activity for health. Geneva: WHO; 2010.

40. Donabedian A. The role of outcomes in quality assessment and assurance. Qual Rev Bull. 1992;18(11):356-60.

41. Donabedian A. Basic approaches to assessment: structure, process and outcome. In: Donabedian A. Explorations in quality assessment and monitoring. Ann Arbor: Health Administration Press; 1980. Vol. 1, p. 77-128.

42. Donabedian A. The seven pillars of quality. Arch Pathol Lab Med. 1990;114(11):1115-8.

\begin{tabular}{r|l} 
ENDEREÇo & \\
Daniel Rogério Petreça & Recebido para publicação: 21/o9/2015 \\
Rua Pascoal Simone, 358 - Coqueiros - 88080-350 & Revisão: 09/04/2018 \\
Florianópolis - SC - BRASIL & Aceito: 11/12/2018 \\
e-mail: profdaniel@unc.com.br &
\end{tabular}

